\title{
Overcoming an intrinsic depolarizing resonance with a partial Siberian snake
}

\author{
H. Huang, ${ }^{1}$ L. Ahrens, ${ }^{1}$ M. Bai, ${ }^{1}$ K. A. Brown, ${ }^{1}$ J.W. Glenn, ${ }^{1}$ A. U. Luccio, ${ }^{1}$ W.W. MacKay, ${ }^{1}$ C. Montag, ${ }^{1}$ V. Ptitsyn, ${ }^{1}$ \\ T. Roser, ${ }^{1}$ N. Tsoupas, ${ }^{1}$ K. Zeno, ${ }^{1}$ V. Ranjbar, ${ }^{2, *}$ H. Spinka, ${ }^{3}$ and D. Underwood ${ }^{3}$ \\ ${ }^{1}$ C-A Department, Brookhaven National Laboratory, Upton, New York 11973, USA \\ ${ }^{2}$ Physics Department, Indiana University, Bloomington, Indiana 47405, USA \\ ${ }^{3}$ Argonne National Laboratory, Argonne, Illinois 60439, USA
}

(Received 8 August 2002; revised manuscript received 19 May 2004; published 27 July 2004)

\begin{abstract}
An $11.4 \%$ partial Siberian snake was used to successfully accelerate polarized protons through a strong intrinsic depolarizing spin resonance in the Alternating Gradient Synchrotron (AGS). No noticeable depolarization was observed. This opens up the possibility of using a $20 \%$ to $30 \%$ partial Siberian snake in the AGS or other medium energy proton synchrotrons to overcome all weak and strong depolarizing spin resonances.
\end{abstract}

DOI: 10.1103/PhysRevSTAB.7.071001

\section{INTRODUCTION}

The high energy spin physics program in the Relativistic Heavy Ion Collider (RHIC) calls for collisions of highly polarized proton beams [1]. Since there is no mechanism for increasing the polarization of protons after they have been injected into RHIC, the level of polarization can only be maximized by increasing the initial polarization of the source and minimizing the depolarization throughout the whole set of accelerators and transfer lines. At present, the level of polarization is limited primarily by depolarizing resonances during the Alternating Gradient Synchrotron (AGS) energy ramp.

Acceleration of polarized proton beams to high energy in circular accelerators is difficult due to numerous depolarizing resonances. During acceleration, a depolarizing resonance is crossed whenever the spin precession frequency equals the frequency with which spin-perturbing magnetic fields are encountered. The spin motion of a particle in circular accelerators is governed by the Thomas-BMT equation [2]. In the presence of the vertical magnetic dipole guide field in a circular accelerator, the spin precesses $G \gamma$ times per orbit revolution, where $G=$ $(g-2) / 2=1.7928$ is the coefficient of the gyromagnetic anomaly of the proton, and $\gamma$ is the Lorentz factor. The number of precessions per revolution is called the spin tune $\nu_{s p}$ and is equal to $G \gamma$ in this case.

There are three main types of depolarizing resonances: imperfection resonances, which are driven by magnet misalignments; intrinsic resonances, driven by the vertical betatron motion through quadrupoles; and coupling resonances, caused by the vertical motion with horizontal betatron frequency due to linear coupling [3]. The resonance condition for an imperfection resonance is $\nu_{s p}=$ $n$, where $n$ is an integer. The resonance condition for an intrinsic resonance is $\nu_{s p}=n P \pm \nu_{y}$, where $n$ is an in-

\footnotetext{
*Present address: Fermi Lab, P.O. Box 500, Batavia, IL 60510, USA.
}

PACS numbers: 41.75.Ak, 07.77.Ka, 29.27.Bd, 29.27.Hj

teger, $P$ is the superperiodicity of the synchrotron, and $\nu_{y}$ is the vertical betatron tune. For the AGS, $P=12$. The resonance condition for a coupling spin resonance is $\nu_{s p}=n \pm \nu_{x}$, where $\nu_{x}$ is the horizontal betatron tune; it is only important if $\nu_{s p}$ is near a strong intrinsic resonance location. When a polarized beam is uniformly accelerated through an isolated spin resonance, the final polarization $P_{f}$ is related to the initial polarization $P_{i}$ by the Froissart-Stora formula [4]

$$
P_{f}=\left(2 e^{-\pi|\epsilon|^{2} / 2 \alpha}-1\right) P_{i},
$$

where $\alpha$ is the resonance crossing rate given by

$$
\alpha=\frac{d\left(\nu_{s p}\right)}{d \theta},
$$

$\epsilon$ is the spin resonance strength [5] and $\theta$ is the orbital bend angle in the synchrotron.

An arrangement of magnets, called a Siberian snake, proposed by Derbenev and Kondratenko [6], can overcome all intrinsic and imperfection depolarizing resonances. A snake rotates the spin of each beam particle by $180^{\circ}$ about an axis in the horizontal plane on each transit without affecting the closed orbit outside of the snake. In a medium energy synchrotron such as the AGS, besides the limited space, a full snake is not practical due to either the large orbit excursion inside a dipole snake or extreme coupling from a solenoidal snake. However, a partial snake which rotates spin by less than $180^{\circ}$ is still feasible. Such a device can overcome all imperfection resonances. At present, the partial snake in the AGS is a solenoid.

For a ring with a partial Siberian snake of strength $s$, the spin tune $\nu_{s p}$ must satisfy the equation [7]

$$
\cos \pi \nu_{s p}=\cos \frac{s \pi}{2} \cos G \gamma \pi,
$$

where the strength $s=1$ corresponds to a full Siberian snake which rotates the spin by $180^{\circ}$. When $s$ is small, the spin tune is nearly equal to $G \gamma$ except when $G \gamma$ is an 
integer $n$, in which case the spin tune $\nu_{s p}$ is shifted away from the integer by $\pm s / 2$ (as shown in Fig. 1). Thus, a partial Siberian snake creates a gap in the spin tune at all integers. Since the spin tune never equals an integer, the imperfection resonance condition is never satisfied. Therefore the partial Siberian snake can overcome all imperfection resonances, provided that the resonance strengths are much smaller than the spin-tune gap created by the partial Siberian snake. An $s=5 \%$ partial Siberian snake [7] has been used to overcome the imperfection resonances in the AGS [8].

Since the intrinsic spin resonance strength is proportional to the betatron amplitude, the final polarization is an ensemble average of the Froissart-Stora formula over the betatron amplitude of the beam particles. A smaller oscillation amplitude corresponds to a weaker spin resonance strength. Therefore, after crossing an intrinsic spin resonance, the spin vector for different particles spreads out, and the beam polarization is decreased. It is difficult to achieve a full spin flip for all particles since the resonance strength of particles in the beam core are small. If the beam is kicked to induce a coherent betatron oscillation so that the betatron oscillation amplitudes of all particles are large, a full spin flip can be attained. A pulsed dipole can induce a coherent betatron oscillation, but it causes emittance growth since this is a nonadiabatic process $[9,10]$. An adiabatic coherent betatron oscillation can be excited and maintained without emittance growth by using an ac dipole magnet operating at a frequency close to a betatron sideband [11]. An artificial spin reso- nance generated by this coherent motion is located at the modulation tune $\nu_{m}$. If we choose the artificial spin resonance location near the intrinsic spin resonance, the spin motion in the vicinity of the intrinsic resonance will be dominated by the artificial spin resonance, and the spin will adiabatically follow the spin closed orbit of the artificial spin resonance [12]. An ac dipole has been used to overcome strong intrinsic resonances in the AGS over the past few years $[12,13]$.

However, the ac dipole technique works only for strong intrinsic resonances, since it relies on the strength of the intrinsic resonances to induce a strong enough artificial resonance. In a medium energy synchrotron such as AGS, there also exist intrinsic resonances which are not strong enough to be overcome by an ac dipole but cause some depolarization with the normal resonance crossing rate. As can be seen from Fig. 1, with a strong enough partial Siberian snake, the spin-tune gap can be increased to allow placing the betatron tune inside the gap so that the intrinsic resonance conditions (both strong and weak) can be avoided. This idea has been proposed previously in Refs. [5,14].

It is worth noting that some $10 \%-20 \%$ partial snake experiments have been done at the low-energy Indiana University Cyclotron Facility Cooler Ring (IUCF) to explore the beam polarization as a function of vertical betatron tune for a partial snake [15]. However, this was done at a fixed energy without acceleration and the partial snake strength was varied to effectively generate the resonance crossing.

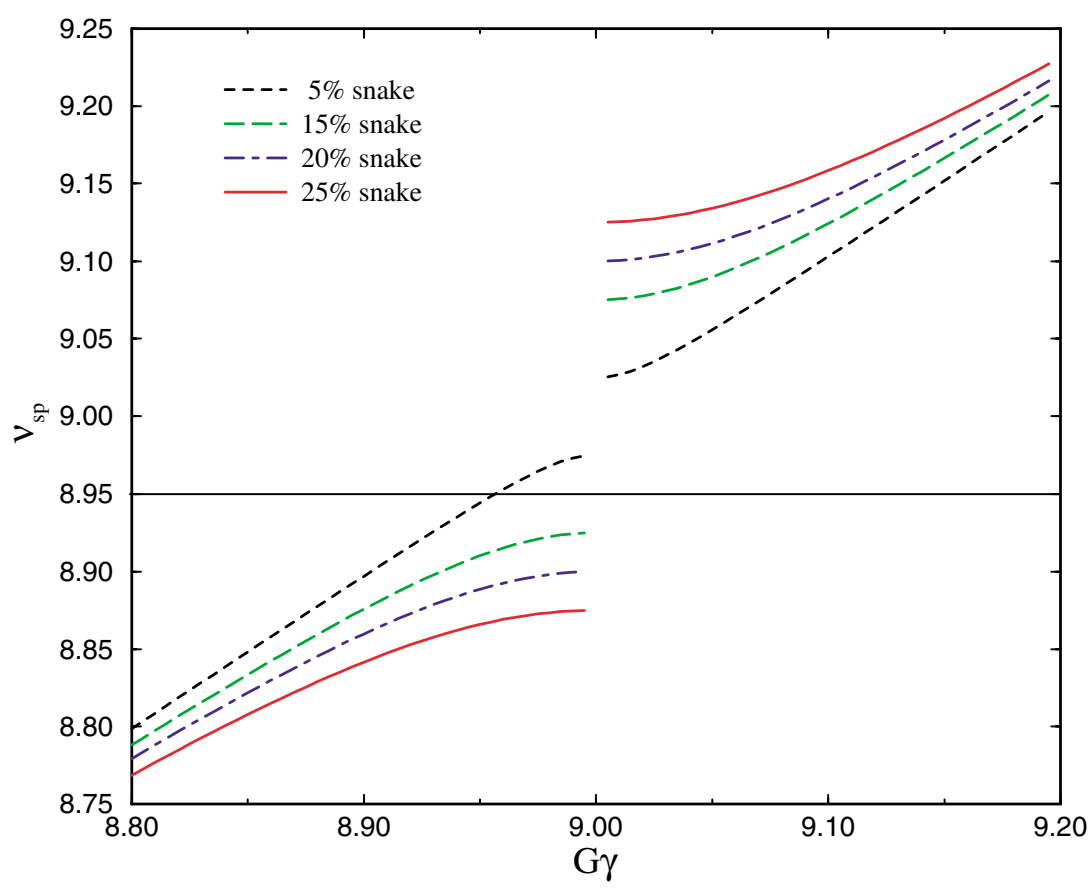

FIG. 1. (Color) Spin tune for various partial Siberian snake strengths. The straight line indicates a possible value for the vertical betatron tune. 
Simulations showed that for the first intrinsic resonance at $0+\nu_{y}$ in the AGS, a $10 \%$ partial Siberian snake would be strong enough to generate the needed spin-tune gap and preserve polarization. In a recent experiment at the AGS this was successfully demonstrated.

\section{EXPERIMENTAL SETUP}

The polarized proton accelerator complex of the Brookhaven AGS and RHIC is shown schematically in Fig. 2. The polarized $\mathrm{H}^{-}$beam from the optically pumped polarized ion source [16] was accelerated through a radio frequency quadrupole and the $200 \mathrm{MeV}$ linac. The beam polarization at $200 \mathrm{MeV}$ was measured with elastic scattering from a carbon fiber target. A fast switching magnet assured the polarization can be measured on subsequent pulses at the end of the linac and in the AGS. During the study, the polarization measured by the $200 \mathrm{MeV}$ polarimeter was $(66 \pm 2) \%$, where the error includes both statistical and systematic uncertainties. The beam was then strip injected and accelerated in the AGS Booster up to $1.5 \mathrm{GeV}$ kinetic energy $(G \gamma=4.7)$. The vertical betatron tune of the AGS Booster was chosen to be 4.9 in order to avoid crossing the intrinsic resonance at $G \gamma=$ $0+\nu_{y}$ in the Booster. The imperfection resonances at $G \gamma=3,4$ in the Booster were corrected by harmonic orbit correctors.

Only one bunch of the $12 \mathrm{rf}$ buckets in the AGS was filled, and the beam intensity varied between 1.3-1.7 $\times$ $10^{11}$ protons per fill. The polarized proton beam was accelerated up to $5.6 \mathrm{GeV}$ kinetic energy $(G \gamma=12.5)$ passing through just one intrinsic resonance located at $G \gamma=0+\nu_{y}$. The resonance crossing rate $\alpha$ was $2.4 \times$ $10^{-5} \mathrm{rad}^{-1}$. Polarization was measured at $G \gamma=12.5$ during a $1 \mathrm{~s}$ flattop after the partial Siberian snake was ramped to zero [17].
The spin rotation angle $\phi$ in the solenoidal snake is given by

$$
\phi=e(1+G) \mu_{0} N I / p,
$$

where $p$ is the momentum of the proton beam, $\mu_{0}$ is the permeability of vacuum, and NI is the current in ampere turns. The effective Siberian snake strength $s$ of the solenoid is $s=\phi / \pi$. The partial snake in the AGS is capable of achieving a 5\% snake strength at $24.2 \mathrm{GeV}$ kinetic energy $(G \gamma=48)$. At $G \gamma=0+\nu_{y}$, the solenoid can in principle generate a $25 \%$ partial snake. However, the solenoidal field will also generate significant coupling, which can cause sizable depolarization. For this experiment an $11.4 \%$ snake strength was chosen as a compromise between obtaining a large enough spintune gap and minimizing the coupling effects. The AGS partial Siberian snake was turned on to $6 \%$ before beam injection into the AGS and then ramped up to $11.4 \%$ before the first intrinsic resonance crossing at $0+\nu_{y}$. The orbit was carefully corrected to maintain beam stability as the vertical betatron tune was moved as high as 8.98. During the experiment, the horizontal betatron tune was kept at 8.54 , while the beam polarization was measured as a function of the vertical betatron tune.

\section{RESULTS AND DISCUSSION}

The experimental data and simulation results are plotted in Fig. 3. The polarization was measured with the AGS internal polarimeter [18]. The errors shown are statistical; there is also an estimated $\pm 12 \%$ systematic uncertainty for the absolute beam polarization calibration at $G \gamma=12.5$. With given vertical and horizontal emittances and known lattice, the resonance strengths for all intrinsic resonances below $G \gamma=12.5$ can be determined. The ratio of polarization level between tune

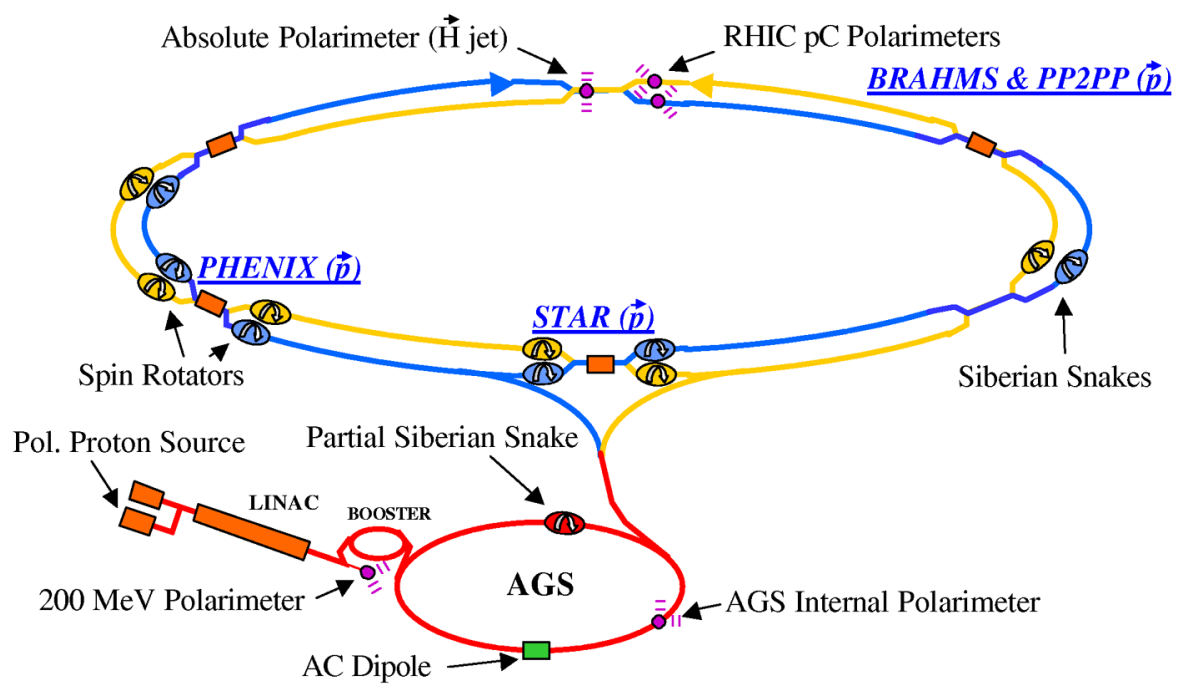

FIG. 2. (Color) The Brookhaven polarized proton facility complex, which includes the optically pumped polarized ion source (OPPIS), $200 \mathrm{MeV}$ linac, the AGS Booster, the AGS, and RHIC. The absolute polarimeter in RHIC will be installed in 2004. 


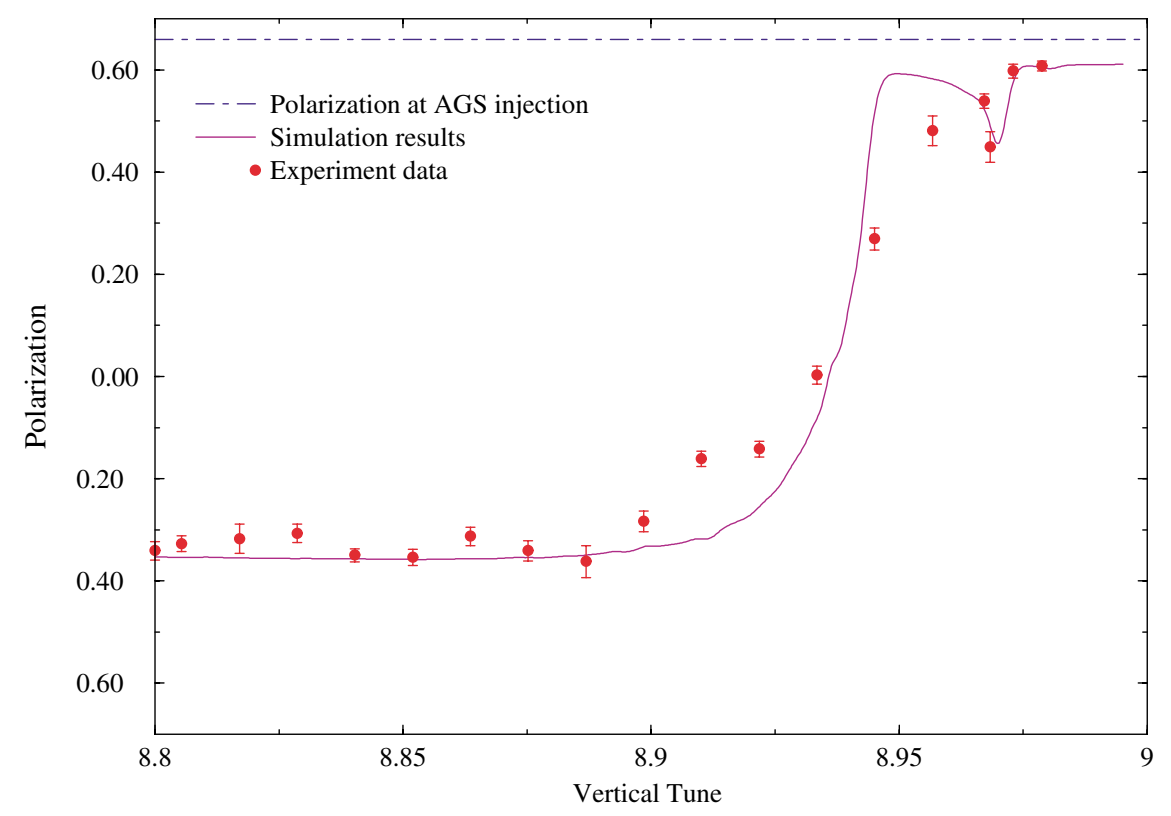

FIG. 3. (Color) The measured vertical polarization as a function of the vertical betatron tune for an $11.4 \%$ partial Siberian snake. The dashed straight line indicates the polarization level measured at the end of the linac. Since the two imperfection resonances in the Booster have been corrected by harmonic orbit correctors, this is also the beam polarization at AGS injection. The solid curve shows the results of combined multiparticle simulations and DEPOL calculations.

settings of 8.80 and 8.98 is determined consequently. The only uncertainty is the possible resonance strength change due to field errors. Calculation shows that the polarization loss can vary only a few percent even with lattice imperfection. This constrains the systematic uncertainty of analyzing power to $\pm 5 \%$. Measured vertical betatron tunes were not available in the tune window $\nu_{y}=$ 8.90 to 8.96. A fit of set tunes to measured tunes outside this window was used to derive the vertical betatron tunes from the set values inside this window. To estimate the depolarizing resonance strength, both vertical and horizontal beam emittances were measured using the AGS ionization profile monitors except for the data points in the tune window $\nu_{y}=8.90$ to 8.96 . As can be seen from Fig. 3, the measured polarization reached a plateau when the vertical betatron tune was very close to 9.00 . The polarization loss in this region was only about $6 \%$ and can be completely explained by spin mismatching at AGS injection and depolarization from coupling resonances as discussed below.

These observations agree well with spin dynamics calculations. With a partial Siberian snake inserted, there are two strong resonances in this energy region: one located at $G \gamma=9$ generated by the partial Siberian snake and the intrinsic resonance at $G \gamma=0+\nu_{y}$. When the intrinsic and artificial resonances do not overlap $\left(\nu_{y} \leq\right.$ 8.85), the resonance at $G \gamma=9$ should flip the spin completely while the intrinsic resonance at $G \gamma=0+\nu_{y}$ causes some depolarization. When the two resonances are very close, such as for $\nu_{y}=8.98$, the intrinsic resonance is overpowered by the resonance at $G \gamma=9$. The particles essentially just experience one resonance at $G \gamma=9$, and full spin flip is observed. When the two resonances are at intermediate separations, such as for $\nu_{y} \approx 8.90$ to 8.95 , they interfere with each other.

A simulation was performed to better understand the polarization behavior in this experiment. The multiparticle tracking is done with two overlapping resonances: one located at $G \gamma=9$ generated by the partial Siberian snake and the other at the intrinsic resonance $G \gamma=0+$ $\nu_{y}$. The strength of the intrinsic resonance was determined from beam size measurements. A vertical tune spread of \pm 0.004 was included in the simulation.

Since there is linear coupling between the beam motion in the two transverse planes, the following coupling resonances in the vicinity of $G \gamma=9$ should also be considered: $G \gamma=17-\nu_{x}, 0+\nu_{x}, 18-\nu_{x}$, and $1+\nu_{x}$. These resonances are not in the spin-tune gap generated by the strong partial snake. Since $\nu_{x}$ and $\nu_{y}$ are well separated, these resonances can be treated separately as isolated resonances. For an isolated depolarizing resonance, its strength can be calculated from the Fourier analysis of the orbit motion for a given lattice. A program called DEPOL [19] has been widely used to calculate the resonance strength for a given emittance and orbit error. The DEPOL program was further extended to include linear coupling effects [20]. We used this extended DEPOL program to calculate the resonance strength. Using the Froissart-Stora formula, the total polarization loss due to the coupling resonances was calculated to be $5 \%$. The polarization loss due to spin mismatching at injection with a $6 \%$ partial Siberian snake was calculated to be 
1\%. The expected polarization from the multiparticle tracking and DEPOL calculations is plotted as the solid curve in Fig. 3.

In general, the simulation agrees well with most data points. The remaining discrepancies for data points between $\nu_{y}=8.90$ and 8.96 could be due to a different beam size or vertical betatron tune, but there were no beam size and tune measurements performed for these data points.

The simulation shows a polarization dip close to $\nu_{y}=$ 8.97, which may also be seen in the experimental data. This is caused by a snake resonance [21] as predicted in Refs. [5,22]. Even when the intrinsic resonance condition cannot be met for $\nu_{y}>8.943$, depolarization can occur from resonance conditions extended over many turns if the intrinsic resonance is very strong. This happens when the following condition is met:

$$
\Delta \nu_{y}=\frac{k \pm \nu_{s p}}{n}
$$

where $\Delta \nu_{y}$ is the fractional part of vertical betatron tune, $n$ and $k$ are integers, and $n$ is called the snake resonance order. The snake resonances have been observed with full snake operation at IUCF [23] and RHIC [24]. With an $11.4 \%$ partial Siberian snake, the fractional part of spin tune is close to 0.057 for $G \gamma \sim 9$. The polarization dip then corresponds to the second order snake resonance $(n=2)$ [25]. With the given resonance crossing rate and intrinsic resonance strength, snake resonances higher than second order do not show a significant effect. The existence of this snake resonance reduces the usable betatron tune space where depolarization is avoided.

At the vertical betatron tune of 8.98 , the difference between the beam polarization at injection and the polarization measured after $0+\nu_{y}$ is due to spin mismatching at injection and depolarization from coupling resonances. If spin matching were achieved at the AGS injection and the linear coupling were eliminated, this scheme could provide full spin flip through the intrinsic resonance. It would also work for weak intrinsic resonances, such as $G \gamma=24 \pm \nu_{y}$, and $48-\nu_{y}$ in the AGS. In addition, if the horizontal betatron tune were also in the gap, the coupling resonance could be avoided.

\section{CONCLUSION}

We have demonstrated for the first time that an $11.4 \%$ partial Siberian snake can effectively overcome an intrinsic depolarizing resonance when the vertical betatron tune is put close to an integer. The critical element of this operation is to maintain beam stability under these conditions. One advantage of this method is that it works for both strong and weak intrinsic resonances. Currently, there is no effective way to overcome the weak intrinsic resonances in the AGS. If the coupling of a new Siberian snake could be reduced, the strength of coupling resonances could also be reduced. Or, if both horizontal and vertical betatron tunes could be put into the spintune gap, both intrinsic and coupling resonances could be avoided.

This is an efficient way to overcome intrinsic resonances in medium energy synchrotrons. A superconducting helical snake with strength on the order of $20 \%$ to $30 \%$ is under construction to replace the current solenoidal AGS partial snake. The helical snake will produce much less coupling than the present weaker solenoidal snake. The study of spin matching at injection and extraction from the AGS is also under way to ensure good polarization transfer efficiency at AGS injection and extraction.

\section{ACKNOWLEDGMENTS}

The research was supported by grants from the U.S. Department of Energy, Division of High Energy Physics Grant No. DE-FG02-92ER40747 and Contract No. W-31109-ENG-38.

[1] Les Bland, in SPIN 2002: 15th International Spin Physics Symposium and Workshop on Polarized Electron Sources and Polarimeters, edited by Y. I. Makdisi, A. U. Luccio, and W.W. MacKay, AIP Conf. Proc. No. 675 (AIP, New York, 2003), p. 98.

[2] L. H. Thomas, Philos. Mag. 3, 1 (1927); J. Frenkel, Z. Phys. 37, 243 (1926); V. Bargmann, L. Michel, and V. L. Telegdi, Phys. Rev. Lett. 2, 435 (1959).

[3] H. Huang, T. Roser, A. Luccio, in Proceedings of the 1997 Particle Accelerator Conference, Vancouver, 1997 (IEEE, Piscataway, NJ, 1997), p. 2538.

[4] M. Froissart and R. Stora, Nucl. Instrum. Methods 7, 297 (1960).

[5] S.Y. Lee, Spin Dynamics and Siberian Snakes in Synchrotrons (World Scientific, Singapore, 1997).

[6] Ya. S. Derbenev and A. M. Kondratenko, Part. Accel. 8, 115 (1978).

[7] T. Roser, in High-Energy Spin Physics, edited by K. J. Heller, AIP Conf. Proc. No. 187 (AIP, New York, 1989), p. 1442.

[8] H. Huang et al., Phys. Rev. Lett. 73, 2982 (1994).

[9] T. Roser, in Proceedings of the 10th International Symposium on High-Energy Spin Physics, Nagoya, Japan, 1992 (Universal Academic Press, Tokyo, 1992), p. 429.

[10] D. A. Crandell et al., Phys. Rev. Lett. 77, 1763 (1996).

[11] M. Bai et al., Phys. Rev. E 56, 6002 (1997); M. Bai, Ph.D. thesis, Indiana University, 1999 (unpublished).

[12] M. Bai et al., Phys. Rev. Lett. 80, 4673 (1998).

[13] M. Bai et al., Phys. Rev. Lett. 84, 1184 (2000).

[14] T. Roser, in Polarized Proton in the AGS Workshop, Upton, NY, 1988 (unpublished).

[15] L.V. Alexeeva et al., Phys. Rev. Lett. 76, 2714 (1996).

[16] A. Zelenski et al., in Proceedings of the 9th International Conference on Ion Sources, Nashville, TN, 2001 [Rev. Sci. Instrum. 73, 888 (2002)]. 
[17] R. A. Phelps et al., Phys. Rev. Lett. 72, 1479 (1994).

[18] C. E. Allgower et al., Phys. Rev. D 65, 092008 (2002).

[19] E. D. Courant and R. D. Ruth, BNL Report No. 51270 (1980).

[20] V. Ranjbar et al., in Proceedings of the 2001 Particle Accelerator Conference, Chicago, 2001 (IEEE, Piscataway, NJ, 2001), p. 3177; V. Ranjbar, Ph.D. thesis, Indiana University, 2002 (unpublished).
[21] S. Y. Lee and S. Tepikian, Phys. Rev. Lett. 56, 1635 (1986); S. Tepikian, Ph.D. thesis, SUNY at Stony Brook, 1986 (unpublished).

[22] S. Y. Lee, Phys. Rev. E 47, 3631 (1993).

[23] R. A. Phelps et al., Phys. Rev. Lett. 78, 2772 (1997).

[24] V. H. Ranjbar et al., Phys. Rev. Lett. 91, 034801 (2003).

[25] C. Ohmori et al., Phys. Rev. Lett. 75, 1931 (1995). 